\title{
ON THE INTERFEROMETRIC AND POLARIMETRIC CAPABILITIES OF THE ARGENTINIAN L-BAND SARAT SYSTEM
}

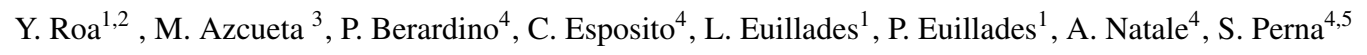 \\ ${ }^{1}$ Universidad Nacional de Cuyo. Facultad de Ingeniería. Instituto CEDIAC\&CONICET, Centro Universitario, 5500 Mendoza, Argentina. \\ (yenni.roa, leonardo.euillades, pablo.euillades)@ingenieria.uncuyo.edu.ar \\ ${ }^{2}$ Instituto de Altos Estudios Espaciales Mario Gulich (CONAE-UNC), Córdoba, Argentina. \\ ${ }^{3}$ Comisión Nacional de Actividades Espaciales, Av. Paseo Colón 751, Buenos Aires, Argentina. (mazcueta@gmail.com) \\ ${ }^{4}$ Istituto per il Rilevamento Elettromagnetico dell'Ambiente del Consiglio Nazionale delle Ricerche (CNR-IREA), \\ Via Diocleziano 328, Naples, Italy. (esposito.c, berardino.p, natale.a)@irea.cnr.it \\ ${ }^{5}$ DI, Università degli Studi di Napoli “Parthenope”, Centro Direzionale Isola C4, Naples, Italy. (perna@ uniparthenope.it )
}

KEY WORDS: SARAT system, airborne SAR, SAR data focusing, Back Projection, Interferometry, Polarimetry

\begin{abstract}
:
In this paper, some results obtained through the processing of data acquired from the Argentinian L-band SARAT system are presented. SARAT is an airborne system that was developed as a first conceptual step toward the design of the Argentinian L- Band satellite mission SAOCOM-1. In particular, some studies relevant to the data focusing, and also regarding the interferometric and polarimetric capabilities of the SARAT system are hereby shown. The considered dataset, relevant to an area located in the Cordoba province, Argentina, has been provided by the National Commission of Space Activities of Argentina (CONAE). This work has been developed in the framework of a collaboration between the Italian Space Agency (ASI) and CONAE.
\end{abstract}

\section{INTRODUCTION}

Synthetic Aperture Radar (SAR) is an active microwave sensor that represents a standard tool for Earth observation (Curlander, McDonough, 1991). The active operating mode makes SAR sensors independent of external radiating sources (e.g., the sunlight), as to guarantee day and night monitoring capabilities. Moreover, the usage of working frequencies belonging to the microwave bands reduces the impact of clouds, fog and rain on the propagation of the transmitted and received signals, thus making SAR systems operative in all-weather conditions.

SAR systems can be mounted onboard different moving platforms, such as satellites (Kramer, 2002) (spaceborne SAR), airplanes (Perna et al., 2016), (Horn et al., 2009), helicopters (Perna et al., 2019) and drones (Ouchi, 2013) (airborne SAR), or also onboard vehicles and ground platforms (Tarchi et al., 2003) (ground based SAR). In particular, spaceborne SAR systems allow the coverage of very wide areas, thus enabling an almost global monitoring of the Earth's surface. However, airborne SAR sensors guarantee a much higher operational flexibility with respect to the spaceborne ones. Indeed, airborne SAR sensors can keep short the revisit time, they allow the prompt monitoring of the area of interest and, unlike spaceborne systems, they can fly practically along any direction. Due to these features, airborne SAR systems represent a very appealing monitoring solution for crisis events (Earthqueakes, landslides, and so on). However, airborne SAR acquisitions are usually affected by motion errors, that is, attitude variations and trajectory deviations, induced on the platform by atmospheric turbulences. Accordingly, good quality Single Look Complex (SLC) images can be obtained by compensating these motion errors through SAR focusing algorithms with integrated MOtion COmpensation (MOCO) procedures (Fornaro, 1999). In particular, the application of MOCO algorithms requires the knowledge of both the flight data and topography information of the illuminated area. The latter is usually provided by an available external Digital Elevation Model (DEM) of the illuminated scene. Flight data, instead, are generally measured onboard the aircraft, by using systems like the Inertial Navigation System (INS) and the Global Positioning System (GPS).

Focusing algorithms integrating MOCO strategies can be implemented in the spectral domain (Fornaro, 1999), (Moreira, Huang, 1994). This allows preserving the high computational efficiency of standard focusing algorithms (Franceschetti, Lanari, 1999) but, at the same time, it requires the usage of some approximations (Fornaro et al., 2006), whose applicability, anyway, is limited to comparatively gentle motion errors. To get accurate focusing results even when radar data are acquired from trajectories with severe motion errors, SAR focusing procedures can be also implemented in time domain (Soumekh, 1999), (Frey et al., 2009). These procedures do not rely on approximations, thus preserving the quality of the SLC images, which is of key interest for most of SAR applications.

In this regard, in last decades a number of techniques based on focused SAR data have been developed and used in many applications for Earth observation and surveillance purposes. In particular, SAR interferometry (InSAR) and Differential InSAR (DInSAR) represent well-established techniques in geodesy and remote sensing: they use two or more SAR images acquired under similar look-angles to generate DEM or deformation maps of the Earth surface (Bamler, Hartl, 1998). Interferometric techniques have been used, for example, to characterize deformation processes associated to volcanic events (Velez et al., 2016) and Earthquakes (Lanari et al., 2010), or for the measurement of ice flows in glaciers (Euillades et al., 2016).

In addition, SAR polarimetry (PolSAR) is a widely used technique which allows combining the information carried by SLC relevant to data acquired at different polarizations, as to build up a powerful information space sensitive to the geometrical (e.g., 
shape) and physical (e.g., permittivity) properties of the scatterers (Lee, Pottier, 2009). Exploiting PolSAR data allows extracting biophysical and geophysical parameters, such as soil moisture, surface roughness (Di Martino et al., 2016), and biomass (Garestier et al., 2009). Of course, interferometric and polarimetric techniques can be implemented by exploiting SAR data acquired at different frequencies. In this regard, in last years worldwide Space Agencies and institutions are showing an increasing interest toward SAR systems operating at the lower bands ( $\mathrm{P}$ and $\mathrm{L}$ ) due to some advantages, such as the larger penetration depths, or the longer decorrelation times (Moreira et al., 2013), that these frequencies exhibit with respect to higher ones ( $\mathrm{C}$ and $\mathrm{X}$ bands). This interest is witnessed by the number of spaceborne SAR missions working at L-band, among which we can mention the already operating systems ALOS2 (JAXA, 2019) of the Japan Aerospace Exploration Agency (JAXA), and SAOCOM 1-A (CONAE, 2019) of CONAE, as well as the forthcoming SAOCOM 1-B (CONAE), TANDEM-L (DLR, 2019b) of the German Aerospace Center (DLR), and NISAR (NASA, 2019a), the joint project between the National Aeronautics and Space Administration (NASA) and the Indian Space Research Organisation (ISRO). Similarly, a number of L-band airborne SAR systems have been developed in last years, as for instance UAVSAR (NASA, 2019b), F-SAR (DLR, 2019a) and SARAT (CONAE). In particular, the SARAT system was developed as a testbed to devise and validate different polarimetric and interferometric applications at L- band, before the launch of SAOCOM-1A.

In this paper, we present some results obtained by processing SARAT data acquired over an area located in Cordoba, Argentina. The main goal of this analysis regards a further investigation of the potentiality of the L-band and a further assessment of the performance of the SARAT system. This work comes from a cooperation between Argentinian and Italian research institutions, in the frame of an ASI-CONAE Agreement.

\section{SYSTEM DESCRIPTION}

The SARAT system was developed by CONAE as a first conceptual step toward the design of the Argentinian L-Band SAR satellite mission SAOCOM-1A. The SARAT project included a full polarimetric L-band airborne SAR sensor and a ground campaign to get in-situ measurements in an agricultural site, useful to evaluate the effectiveness of SAR data for the retrieval of soil parameters. The SAR system was installed on a Beechcraft Super King Air B-200 (Figure 1) of the Armada de la República Argentina (ARA). The aircraft has a nominal range of flight altitudes between 4000 and 6000 meters above the Earth's surface (Solorza et al., 2013). The main characteristics of the SARAT system are summarized in Table 1.

\section{DATA SET}

The National Commission of Space Activities of Argentina has a catalog for internal use where, among others, are stored SARAT data. In particular, within the available SARAT dataset, we selected eight tracks (hereafter labeled as 19, 22, 29, $33,43,50,53,69)$ flown by the SARAT system over the same scene to evaluate the possibility of performing interferometric and polarimetric analyses.

The data were acquired on March 2010 and cover an area including the Teófilo Tabanera Space Center (CETT) of CONAE,

\begin{tabular}{|l|c|}
\hline Central frequency & $1.3 \mathrm{GHz}$ \\
Chirp bandwidth & $38 \mathrm{MHz}$ \\
Pulse duration & $10 \mu \mathrm{s}$ \\
Pulse Repetition Frequency & $250 \mathrm{~Hz}$ \\
ADC sampling frequency & $50 \mathrm{MHz}$ \\
Antenna pointing angle & $45^{\circ}$ \\
Antenna size & $1.8 \times 0.30 \mathrm{~m}$ \\
Flight speed & $100 \mathrm{~m} / \mathrm{s}$ \\
Polarization & Quad-Pol \\
Look angle & $20^{\circ}-70^{\circ}$ \\
Swath & $12 \mathrm{Km}$ \\
Azimuth resolution & $0.9 \mathrm{~m}$ \\
Range resolution & $3.9 \mathrm{~m}$ \\
Nominal range of flight heights & $4-6 \mathrm{Km}$ \\
\hline
\end{tabular}

Table 1. Characteristics of the SARAT system.

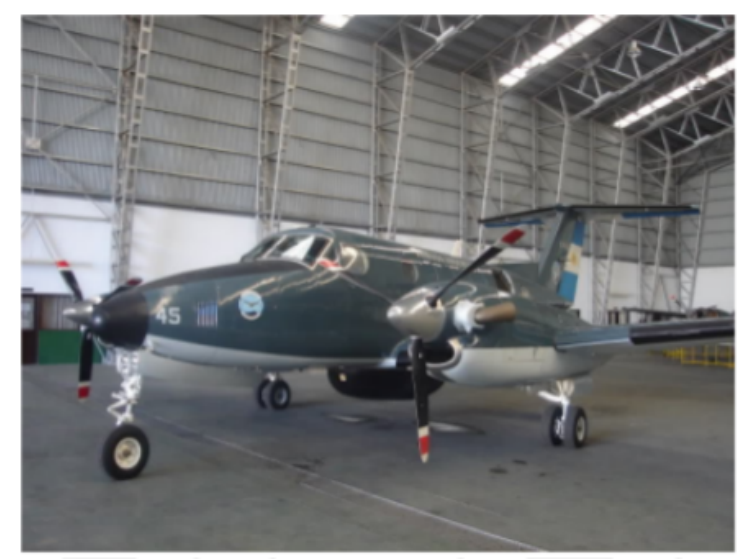

(a) Aircraft of the ARA.

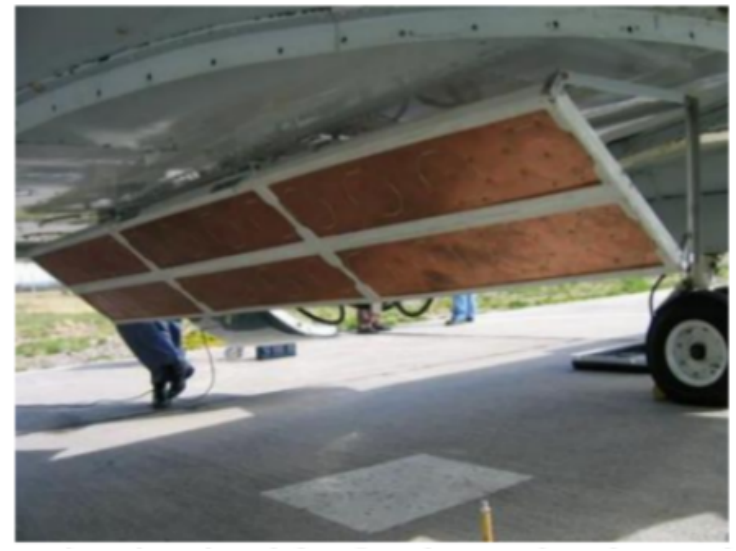

(b) Antenna of the SARAT system

Figure 1. The SARAT system (Notarnicola, Solorza, 2014).

situated in the Cordoba province, Argentina (see Figure 2). Selected data (ARD, which stands for Raw Data Annotated) provided by CONAE represent Level 0 products and were obtained from the binary data flow (Raw Data). For every track the system collected data in the four polarization channels $(\mathrm{HH}$, $\mathrm{VV}, \mathrm{HV}$ and $\mathrm{VH}$ ).

Generally, focusing airborne SAR data requires the availability of precise navigation data, the accurate knowledge of the lever arm (the distance between the SAR antenna and the GPS system) and the accurate knowledge of the orientation angle of the INS. In this regard, GPS and INS data relevant to the considered tracks were also provided by CONAE. 


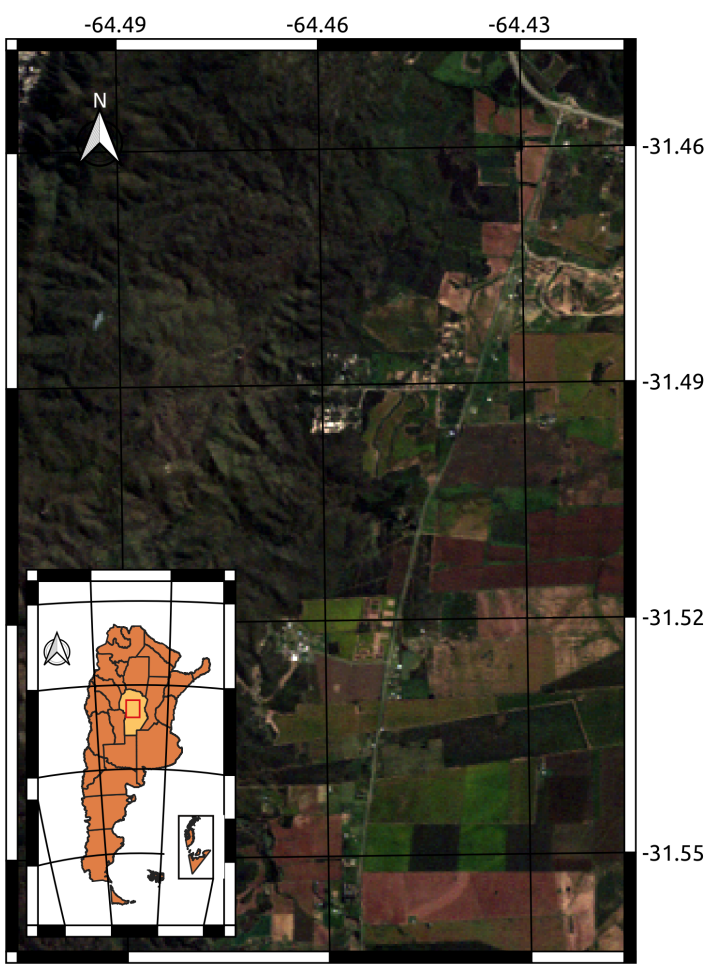

Figure 2. Test site area which includes the Teofilo Tabanera Space Center (CETT) of CONAE, situated in the Cordoba province, Argentina.

\section{RESULTS}

In this Section we show some results relevant to the focusing of the considered SARAT data. Moreover, here we also provide the interferometric and polarimetric analyses which have been carried out on the focused dataset. Data focusing has been carried out through a time domain Back Projection (BP) algorithm (Soumekh, 1999), (Frey et al., 2009), according to the processing settings listed in Table 2. This allowed us to focus the whole considered dataset over the same output grid.

\begin{tabular}{|l|c|}
\hline Azimuth resolution & $3 \mathrm{~m}$ \\
Range resolution & $3.95 \mathrm{~m}$ \\
Range pixel spacing & $3 \mathrm{~m}$ \\
Azimuth pixel spacing & $0.4 \mathrm{~m}$ \\
Range size & $10 \mathrm{Km}$ \\
Azimuth size & $5 \mathrm{Km}$ \\
\hline
\end{tabular}

Table 2. Main processing settings for the considered focused SARAT images.

Some defocusing effects have been observed in the obtained images. First, from the analysis of the SLC images in correspondence of a trihedral corner reflector (CR) deployed in the test area, we observed the broadening of the point spread function of the system. This effect turned out to be independent of the polarization of the transmitted and received signals. An example of this effect is shown in Figure 3, where we depict the SLC image (HH polarization) relevant to track 19 (Figure 3(a)), along with its azimuth cut in correspondence of the CR (Figure 3(b)).

Second, we found a relative stretching between SLCs relevant to different tracks. This effect is shown in Figure 4 (left and center panels) where we depict the SLCs (HH polarization) rel-

evant to the tracks 19 and 33. In this regard we recall that both images have been focused on the same output grid, and thus, the two SLCs are expected to be perfectly overlapping; this however does not happen in wide areas of the images such as those highlighted with red circles in the figure. Both the effects are due to the presence of residual uncompensated motion errors (Fornaro et al., 2005), which reasonably arise from some inaccuracies of the navigation data.

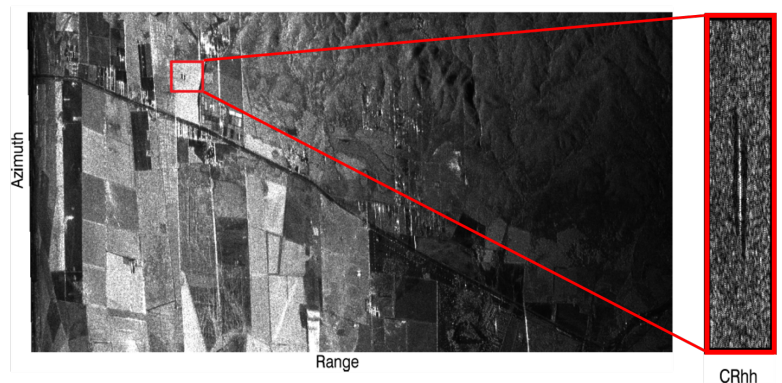

(a)

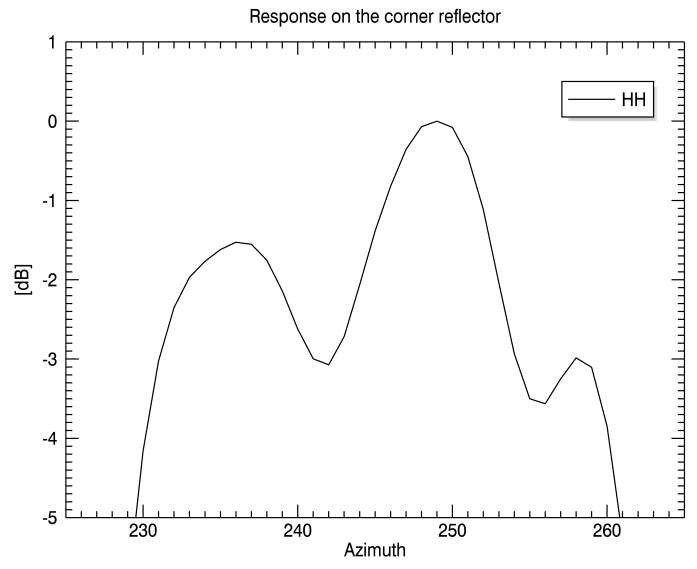

(b)

Figure 3. Broadening effect observed in the SLC relevant to track 19. a) SLC amplitude image (HH polarization) relevant to track 19. Red rectangles highlight the region surrounding the corner reflector. b) Azimuth cut of the SAR response over the corner reflector.

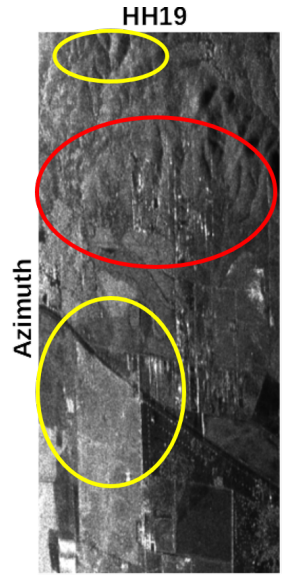

Range

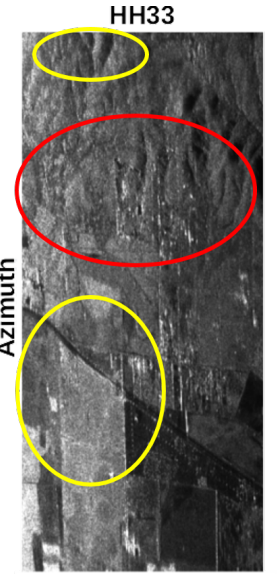

Range

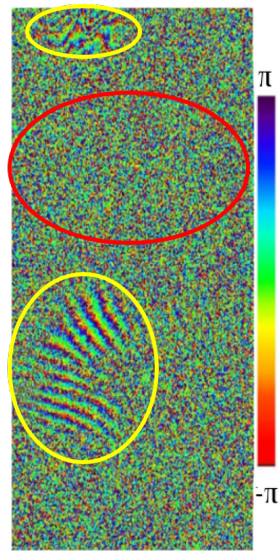

Figure 4. SLC amplitude images relevant to the tracks 19 and 33 and the resulting interferogram. 
Of course, the quoted geometric distortions affect the generation of repeat-pass InSAR products achievable through the SARAT system. This is shown in the right panel of Figure 4, where we depict the interferogram relevant to the SLCs reported in the same figure. From the interferogram, we can observe the presence of interferometric fringes only in the areas (higlighted in yellow) where the SLCs well overlap each other. As matter of fact, the quality of the repeat-pass InSAR products can be improved by applying a further co-registration algorithm based on the cross-correlation of SLC data. To show this, in Figure 5 we show the SLCs relevant to the track 22 (left panel) and track 19 (center panel), and the SLC relevant to track 22 after the application of the quoted co-registration algorithm (right panel). It is stressed once again that with the used focusing strategy the images on the left panel and on the center panel are expected to be already overlapping each other. Moreover, in Figure 6 we show the obtained interferograms before (left panel) and after (right panel) applying the co-registration algorithm.

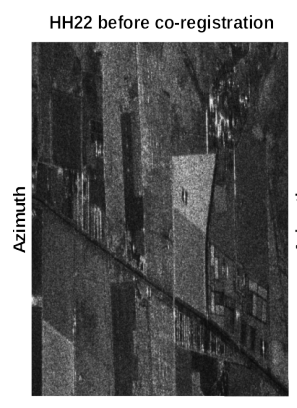

Range

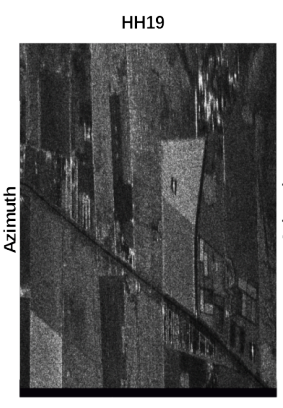

Range

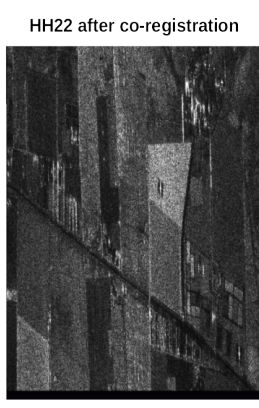

Range
Figure 5. SLC amplitude images (HH polarization) relevant to the tracks 19 and 22 before (left panel) and after (right panel) applying the co-registration algorithm.
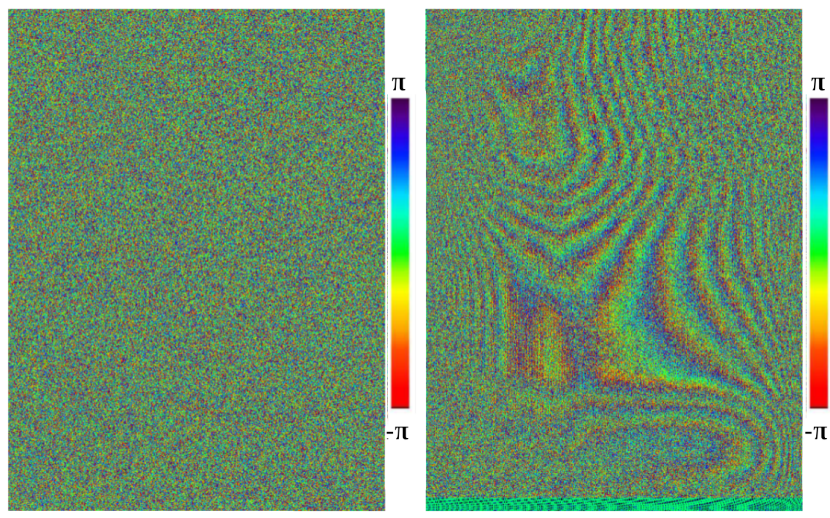

Figure 6. Interferogram between HH19 and HH22 before (on the left) and after (on the right) applying the co-registration step.

With regard to the polarimetric capabilites of the SARAT system, we first of all evaluated the need to calibrate the available quad-pol data. To this aim, we checked if some properties of the polarimetric scattering from canonical objects were respected from the measured data. We considered, among others, some properties relevant to the phase of the co-polar (i.e., HH/VV) correlation coefficient. It is indeed well known that its value over trihedral corner reflectors (odd-bounce scattering) must be close to $0^{\circ}$ (Van Zyl, 1990). Moreover, the difference between its values over a $\mathrm{CR}$ and over a building (even-bounce scattering) must be close to $\pm 180^{\circ}$ (Van Zyl, 1989). We carried out this analysis over two areas including the CR and a building of the CETT, and we realized that a polarimetric calibration step, aimed at removing the cross-talk and channel imbalance effects, was required. Therefore, an automatic procedure for polarimetric calibration based on the Quegan algorithm (Quegan, 1994) has been developed and applied to the considered SARAT data. In Figure 7 we show the phase of the co-polar correlation coefficient obtained by considering the calibrated SARAT data acquired along track 19. It can be observed that this phase exhibits a greenish appearance (i.e., a value of about $2^{\circ}$ ) in correspondence of the CR highlighted in the yellow circle (see also Figure 8), and a violetish appearance (i.e., a value of about $175^{\circ}$ ) in correspondence of a building of the CETT highlighted in the white circle, thus fulfilling the above mentioned properties of the polarimetric scattering.

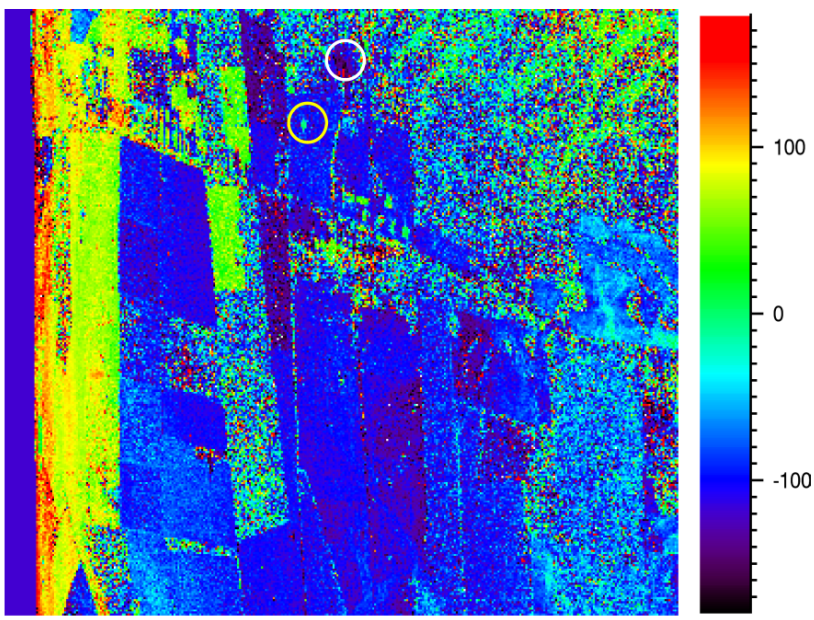

Figure 7. Phase of the co-polar correlation coefficient for the calibrated SARAT data acquired along track 19.
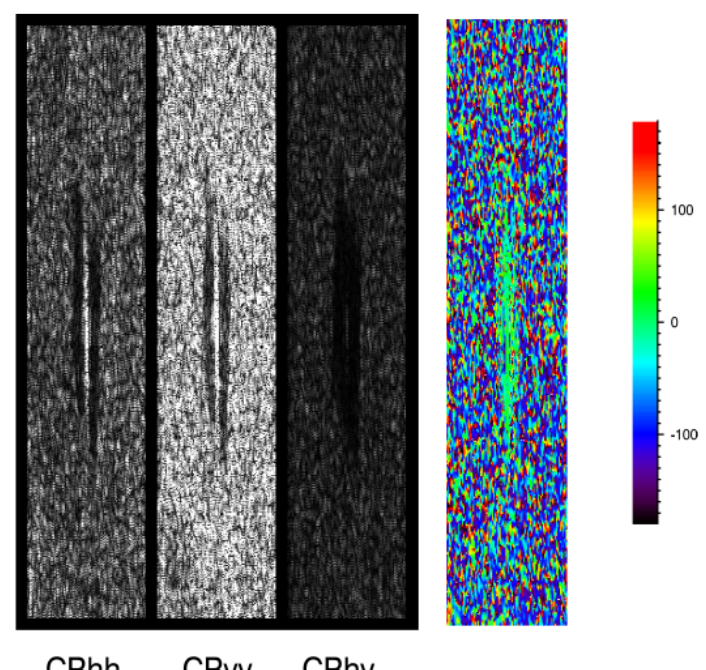

CRhh CRvv CRhv

Figure 8. SLC amplitude images (HH,VV and HV polarizations) and phase image of the co-polar correlation coefficient for the calibrated SARAT data acquired along track 19 and relevant to the CR.

After the calibration step, a preliminary study has been carried 
out to evaluate the capabilities of the polarimetric SARAT data for land classification purposes. To this aim, we considered the Pauli decomposition to identify the dominant scattering mechanism for each target of the observed scene (Lee, Pottier, 2009). In Figure 9 we show the color-coded Pauli map relevant to the calibrated SARAT data acquired along track 19. From the this
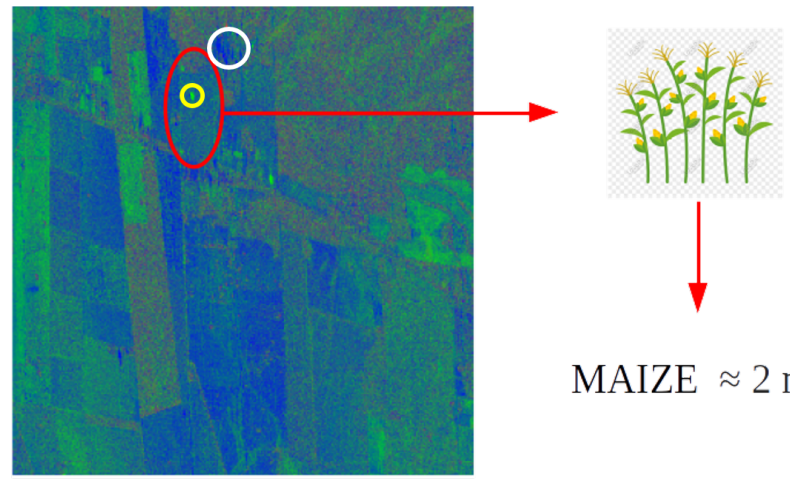

MAIZE $\approx 2 \mathrm{~m}$

Figure 9. Pauli decomposition for the calibrated SARAT image relevant to track 19. Red: volume. Green: surface. Blue: double-bounce.

map we can observe that the dominant scattering mechanisms are properly identified for both the CR (in the yellow circle) and the CETT building (in the white circle). Moreover, a further correspondence between the ground truth and the classification results provided by the quoted Pauli map can be found in the region surrounding the $\mathrm{CR}$, characterized by a dominant evenbounce scattering mechanism. This result is in agreement with what reported by CONAE, since this region corresponded to a maize field, where the vegetation was in an advanced stage of growth (i.e., with a height of about $2 \mathrm{~m}$ ) at the SAR acquisition time.

\section{CONCLUSION}

In this work we presented some results of the activities carried out at IREA-CNR, in Italy, in the framework of a collaboration ASI-CONAE. In particular, we showed some processing results obtained by using a dataset (SAR raw data, IMU data and GPS data) acquired over Cordoba, Argentina, by the Argentinian SARAT system on March 2010. The SARAT system is not currently operative. First of all, we presented an analysis related to the focusing of the raw data. In this regard, two defocusing effects, namely a broadening of the point spread function and a geometric relative stretching among SLCs relevant to different tracks, were found. Both these effects can be linked to the inaccuracy of the available navigation data. In addition, we presented some interferometic results by showing that the quoted stretching effects affect the generation of repeat-pass InSAR products, whose quality, however, can be improved by exploiting proper co-registration procedures. It is remarked that these co-registration procedures would be useless in absence of residual motion errors, since the adopted focusing procedure allows us to generate all the SLC images on the same output grid. Finally, we provided some results relevant to the analysis of the full-polarimetric data acquired by the system. In this regard, we have shown the need of applying a polarimetric calibration to the considered dataset and, moreover, we have also presented some classification results based on the Pauli decomposition.

\section{ACKNOWLEDGEMENTS}

This work was supported by Universidad Nacional Cuyo with the project SIIP 06/B367 Res. 4142/2019-R. This study was also supported by the Ministero degli Affari Esteri e della Cooperazione Internazionale of Italy, the Agenzia Spaziale Italiana (ASI) and Instituto Gulich (CONAE-UNC). The Authors express their gratitude to CONAE for providing the considered SARAT dataset and to ASI for the scholarship grant that supported the cooperation between the researchers of the Argentinian and Italian institutions. Moreover, the Authors also thank Álvaro Soldano and Ezequiel De Luca from CONAE for their support in the pre-processing steps of SARAT data.

\section{REFERENCES}

Bamler, R., Hartl, P., 1998. Synthetic Aperture Radar Interferometry. Inverse problems, 14(4), R1.

CONAE, 2019. Misiones Satelitales. SAOCOM, 2019. https://www . argentina.gob.ar/ciencia/conae/ misiones-espaciales/saocom.

Curlander, J., McDonough, R., 1991. Synthetic Aperture Radar - Systems and Signal Processing. John Wiley Sons, New York.

Di Martino, G., Iodice, A., Natale, A., Riccio, D., 2016. Polarimetric Two-Scale Two-Component Model for the Retrieval of Soil Moisture Under Moderate Vegetation via L-Band SAR Data. IEEE Transactions on Geoscience and Remote Sensing, 54, 2470-2491.

DLR, 2019a. F-SAR - The New Airborne SAR System. FSAR, 2019. https://www.dlr.de/hr/en/desktopdefault. aspx/tabid-2326/3776_read-5691/.

DLR, 2019b. Tandem-L. A Satellite Mission for Monitoring Dynamic Processes on the Earth's Surface. TandemL, 2019. https://www.dlr.de/hr/en/desktopdefault. aspx/tabid-8113/14171_read-35837/.

Euillades, L. D., Euillades, P. A., Riveros, N. C., Masiokas, M. H., Ruiz, L., Pitte, P., Elefante, S., Casu, F., Balbarani, S., 2016. Detection of glaciers displacement time-series using SAR. Remote Sensing of Environment, 184, 188-198.

Fornaro, G., 1999. Trajectory deviations in airborne SAR: analysis and compensation. IEEE Transactions on Aerospace and Electronic Systems, 35(3), 997-1009.

Fornaro, G., Franceschetti, G., Perna, S., 2006. On center-beam approximation in SAR motion compensation. IEEE Geoscience and Remote Sensing Letters, 3(2), 276-280.

Fornaro, G., G.Franceschetti, Perna, S., 2005. Motion compensation errors: effects on the accuracy of airborne SAR images. IEEE Transactions on Aerospace and Electronic Systems, 41(4), 1338-1352.

Franceschetti, G., Lanari, R., 1999. Synthetic Aperture Radar Processing. CRC press, New York.

Frey, O., Magnard, C., Ruegg, M., Meier, E., 2009. Focusing of airborne Synthetic Aperture Radar data from highly nonlinear flight tracks. IEEE Transactions on Geoscience and Remote Sensing, 47(6), 1844-1858. 
Garestier, F., Dubois-Fernandez, P., Guyon, D., Le Toan, T., 2009. Forest Biophysical Parameter Estimation Using L and P band Polarimetric SAR data. IEEE Transactions on Geoscience and Remote Sensing, 47, 3379 - 3388.

Horn, R., Nottensteiner, A., Reigber, A., Fischer, J., Scheiber, R., 2009. F-SAR - DLR's new multifrequency polarimetric airborne SAR. IEEE International Geoscience and Remote Sensing Symposium 2009, 902-905.

JAXA, 2019. Advanced Land Observing Satellite. ALOS2, 2019. https://www.eorc.jaxa.jp/ALOS-2/en/about/ palsar2.htm.

Kramer, H. J., 2002. Observation of the Earth and Its Environment. Survey of Missions and Sensors. Springer.

Lanari, R., Berardino, P., Bonano, M., Casu, F., Manconi, A., Manunta, M., Manzo, M., Pepe, A., Pepe, S., Sansosti, E., Solaro, G., Tizzani, P., Zeni, G., 2010. Surface displacements associated with the L'Aquila $2009 \mathrm{Mw} 6.3$ earthquake (central Italy): New evidence from SBAS-DInSAR time series analysis. Geophysical Research Letters, 37(20), 1-6.

Lee, J., Pottier, E., 2009. Polarimetric Radar Imaging: From Basics to Applications. CRC Press, New York.

Moreira, A., Huang, Y., 1994. Airborne SAR processing of highly squinted data using a chirp scaling approach with integrated motion compensation. IEEE Transactions on Geoscience and Remote Sensing, 32(5), 1029-1040.

Moreira, A., Prats-Iraola, P., Younis, M., Krieger, G., Hajnsek, I., Papathanassiou, K., 2013. A tutorial on Synthetic Aperture Radar. IEEE Geoscience and Remote Sensing Magazine, 3, 138.

NASA, 2019a. NASA-ISRO SAR Mission (NISAR). NISAR, 2019. https://nisar.jpl.nasa.gov/.

NASA, 2019b. UAVSAR. Uninhabited Aerial Vehicle Synthetic Aperture Radar. UAVSAR, 2019. https://uavsar. jpl.nasa.gov/.

Notarnicola, C., Solorza, R., 2014. Integration of remotely sensed images and electromagnetic models into a bayesian approach for soil moisture content retrieval: methodology and effect of prior information. Dynamic Programming and Bayesian Inference, Concepts and Applications, IntechOpen.

Ouchi, K., 2013. Recent Trend and Advance of Synthetic Aperture Radar with Selected Topics. Remote Sensing, 716 - 807.

Perna, S., Alberti, G., Berardino, P., Bruzzone, L., Califano, D., Catapano, I., Ciofaniello, L., Donini, E., Esposito, C., Facchinetti, C., Formaro, R., Gennarelli, G., Gerekos, C., Lanari, R., Longo, F., Ludeno, G., Mariotti d'Alessandro, M., Natale, A., Noviello, C., Palmese, G., Papa, C., Pica, G., Rocca, F., Salzillo, G., Soldovieri, F., Tebaldini, S., Thakur, S., 2019. The ASI Integrated Sounder-SAR System Operating in the UHFVHF Bands: First Results of the 2018 Helicopter-Borne Morocco Desert Campaign. Remote Sensing, 11, 1845.

Perna, S., Esposito, C., Amaral, T., Berardino, P., Jackson, G., Moreira, J., Pauciullo, A., Vaz Junior, E., Wimmer, C., Lanari, R., 2016. The InSAeS4 airborne X-band interferometric SAR system: A rst assesment on its imaging and topographic mapping capabilities. Remote Sensing, 8, 40.
Quegan, S., 1994. A unified algorithm for phase and cross-talk calibration of polarimetric data-theory and observations. IEEE Transactions on Geoscience and Remote Sensing, 32(1), 89-99.

Solorza, R., Notarnicola, C., Karszenbaum, H., 2013. Retrieval of soil moisture using electromagnetic models and a bayesian approach in view of the saocom mission: Study on sarat images in an agricultural site in argentina. 2013 IEEE International Geoscience and Remote Sensing Symposium - IGARSS, 739-742.

Soumekh, M., 1999. Synthetic Aperture Radar Signal Processing with MATLAB Algorithms. John Wiley Sons, Inc., New York.

Tarchi, D., Casagli, N., Fanti, R., Leva, D., Luzi, G., Pasuto, A., Pieraccini, M., Silvano, S., 2003. Landslide monitoring by using ground-based SAR interferometry: An example of application to the Tessina landslide in Italy. Eng. Geol., 68, 15-30.

Van Zyl, J. J., 1989. Unsupervised classification of scattering behavior using radar polarimetry data. IEEE Transactions on Geoscience and Remote Sensing, 27(1), 36-45.

Van Zyl, J. J., 1990. Calibration of polarimetric radar images using only image parameters and trihedral corner reflector responses. IEEE Transactions on Geoscience and Remote Sensing, 28(3), 337-348.

Velez, M., Euillades, P., Blanco, M., Euillades, L., 2016. Ground deformation between 2002 and 2013 from insar observations. Copahue volcano, Springer, 175-198. 